\title{
Le choix professionnel passe avant le numerus clausus
}

\section{Yvonne Gilli}

Dr méd., membre du Comité central de la FMH, responsable du département Numérisation / eHealth

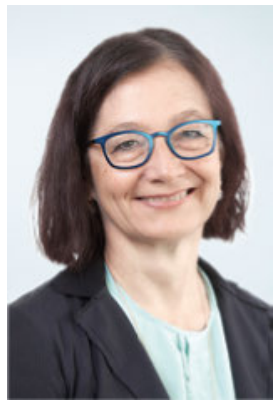

Une fois de plus, le Parlement s'est penché sur différents critères pour l'admission aux études de médecine. Dans une motion (Mo. 15.3687), la conseillère nationale Ruth Humbel (PDC) demande au Conseil fédéral d'examiner la possibilité d'une autre formule pour l'examen du numerus clausus actuel et d'introduire un stage pour remplacer voire compléter les examens portant sur les aptitudes cognitives. Cette motion a été approuvée à une large majorité par le Conseil national. Le nouveau conseiller fédéral et médecin Ignazio Cassis s'est abstenu. Toutefois, lui aussi avait demandé en 2015 le réexamen du numerus clausus en se référant au modèle israélien (Po. 15.3967). Ensemble, ils avaient soutenu une procédure de sélection tenant également compte des compétences sociales et de l'intelligence émotionnelle en plus des aptitudes cognitives, mais leur proposition n’a eu aucune chance auprès de la Commission de la science, de l'éducation et de la culture du Conseil des Etats, qui a suivi les arguments des délégués de la Conférence suisse des hautes écoles et de la Conférence des recteurs (swissuniversities). En plus des considérations fédéralistes, ces derniers ont avancé l'argument des coûts des places de stage et la subjectivité des critères d'évaluation.

Hänsgen et al. du Centre pour le développement de tests et diagnostic de l'Université de Fribourg résument bien, sous un angle certes plutôt positif, le numerus clausus. Selon eux, le critère économique a constitué un atout de taille lors de l'introduction du numerus clausus en Suisse grâce à la possibilité d'utiliser le test allemand TMS à des conditions avantageuses. Les entretiens et les stages pratiques s'étaient révélés trop coûteux. Avec le numerus clausus, les candidats qui ont les meilleures chances de terminer leurs études dans un temps relativement bref et avec les meilleures prestations sont admis en priorité. Le test examine l'aptitude aux études (output) et non l'aptitude professionnelle (outcome).

Les personnes qui se présentent à l'examen sont toutefois des personnes ayant fait un choix professionnel. Il existe très peu d'études sur la motivation conduisant au choix de la profession de médecin. Nous savons que les étudiants en médecine sont avant tout motivés par des idéaux sociaux («j'ai envie de travailler avec les gens», «d'aider les gens») et par une curiosité scientifique («activité intellectuellement exigeante»), qui sont les principaux facteurs les ayant conduits à faire ce choix. Sur le plan sociodémographique, on remarque que les parents ont, en moyenne, plus souvent une formation académique et/ou qu'ils sont eux-mêmes médecins (Belastungserleben, Albrecht, 2016).

Comme argument en faveur du numerus clausus, Hänsgen et al. mentionnent qu'une sélection intra-universitaire au moyen d'un examen après la première année représente d'une part une perte de temps pour les étu-

\section{Le test (numerus clausus) examine l'aptitude aux études et non l'aptitude à la profession.}

diants qui échouent en raison des taux d'échec élevés, et attise d'autre part la concurrence, ce qui «risque fort de développer chez les étudiants des traits de caractère peu favorables.» Tandis que le premier argument peut également être reproché au numerus clausus, ce dernier argument paraît relativement cynique sachant que les évaluations par le personnel soignant montrent un net potentiel d'amélioration dans la collaboration interdisciplinaire avec les médecins (Interprofessionelle Zusammenarbeit, Schärli, 2017). Pour l'exploiter, davantage de compétences sociales et d'intelligence émotionnelle sont requises, avant tout du côté des médecins.

Les milieux politiques, les instances universitaires et la FMH s'accordent tous sur la nécessité de réglementer les admissions. Il y a peu de place pour les approches intégratives, qui vont du choix motivationnel intrinsèque pour une profession à la conception d'études apprenant à être un «bon médecin». Lors l'audition sur la motion Humbel au Conseil des Etats, la FMH a défendu une procédure d'admission en deux phases venant compléter le numerus clausus par des tests de jugement situationnel, des multiple mini-interviews ou des stages. 